\title{
Michael Grunstein and David Allis receive the 2018 Lasker Basic Medical Research Award
}

$T_{1}$ he sequence of an individual's genome provides basic instructions for development and determines many phenotypic traits; however, sequence alone does not account for all variation in gene expression patterns among diverse cell types and individuals. It is now recognized that modification of DNA-associated proteins and complexes is critical for determining how and when genes are expressed, transferred, and copied. Histone alterations have been linked to multiple diseases and have become attractive therapeutic targets. The 2018 Lasker Basic Medical Research Award honors Michael Grunstein of UCLA and David Allis of The Rockefeller University (Figure 1) for their contributions toward unravelling the essential role of histones and histone modification in gene regulation.

\section{More than a pretty package}

Histones were first described in 1884 by Albrecht Kossel. This new set of proteins was isolated via acid extraction and closely associated with nucleic acids. Despite their early identification, histones were largely overlooked until the 1960s, at which time multiple histone types (H1, H2A, H2B, H3, and H4) were identified. Several experiments indicated that chromatin may harbor a repeating structure. In 1974, Kornberg first proposed an octameric particle consisting of the $\mathrm{H} 3-\mathrm{H} 4$ tetramer and two H2A-H2B dimers around which approximately 200 bp of DNA was wrapped. It is now known that $147 \mathrm{bp}$ of DNA wraps around each histone octamer to form the nucleosome and that linker DNA between nucleosomes accounts for the noted "beads on a string" appearance of chromatin in the electron microscope. Histone protein $\mathrm{H} 4$ was shown to be almost invariant among widely divergent species (peas and cows), testifying to the importance of the histone in eukaryotic evolution; however, the function of histones was not clear (see refs. 1 and 2 for reviews). The
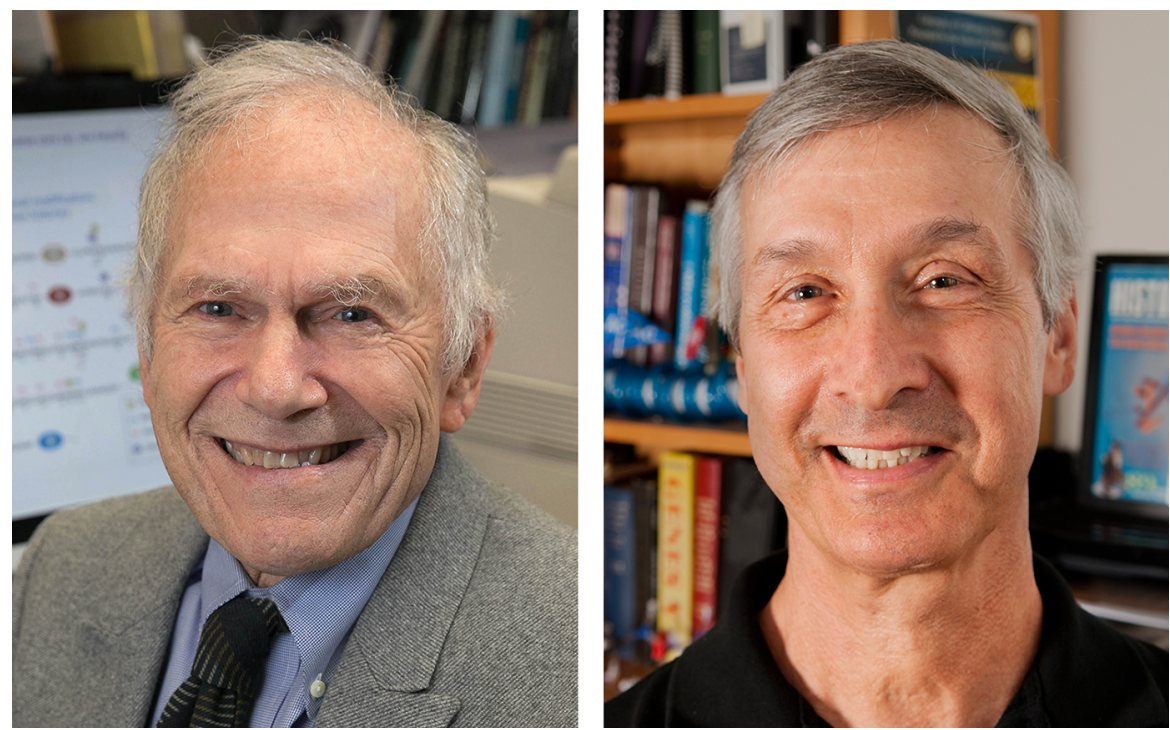

Figure 1. The recipients of the 2018 Lasker Basic Medical Research Award. Michael Grunstein (left) of UCLA and David Allis (right) of The Rockefeller University are being recognized for their work toward elucidating the role of histones and histone modification in gene expression. Photo of Michael Grunstein courtesy of Reed Hutchinson/UCLA Health. Photo of David Allis courtesy of The Rockefeller University.

nucleosome was widely viewed to solely function to tightly package DNA and not to be involved in DNA regulation; however, in 1987, Lorch and Kornberg showed that nucleosomes were able to inhibit the initiation of gene transcription in vitro (3). Then, in 1988, Michael Grunstein and his graduate student Min Han provided the first in vivo demonstration of histonedependent gene regulation (4). Grunstein and Han generated strains of yeast with the sole histone $\mathrm{H} 4$ gene under control of the GAL promoter, a glucose-responsive promoter. When these strains were shifted to glucose, $\mathrm{H} 4$ transcription was suppressed, thereby depleting nucleosomes from the cells as their DNA continued to replicate one last time. The loss of nucleosomes resulted in expression of genes normally repressed in the presence of glucose; thus, nucleosomes themselves were required for gene repression.

This exciting demonstration of histonedependent alteration of gene expression in vivo was made possible by the use of yeast as a model system. Grunstein had first become interested in histones due to their repetitive nature, much like the ribosomal genes that he helped isolate during his time as a graduate student at the University of Edinburgh in Max Birnstiel's lab. He pursued his interest in histone-encoding mRNAs during his postdoctoral training with Larry Kedes at Stanford University. At that time, the focus was on histone-encoding mRNAs in the sea urchin, the genome of which contained approximately 400 copies of the histone genes. Grunstein went on to start his own lab at UCLA, where he planned to continue his work on histones in the sea urchin; however, several obstacles led to a change of model. As Grunstein recently recalled to the JCI, "I spent two years as an assistant professor trying to get urchins from the Pacific. There were El Niños at that time, and we didn't have any significant material for close to two years. As a new assistant professor, I only had a limited time to get things to work and began looking for another system." Grunstein soon became interested in yeast, which have only 2 copies of each of the histone genes. Grunstein credits the shift in model organisms to two graduate students 
in his lab and an inspirational course at Cold Spring Harbor. "I had two wonderful graduate students, Mary Rykowski and John Wallis, who started the yeast work in the lab while I went to Cold Spring Harbor to take the yeast genetics course." Histones contain highly acetylated lysines at their $\mathrm{N}$ termini and were shown to correlate with increased gene activity (5); however, it was not clear whether acetylation was a cause or result of transcription. Grunstein and his postdoc Linda Durrin would also go on to show that acetylation sites on $\mathrm{H} 4$ were required for gene activation (6); however, the enzymes responsible for acetylating $\mathrm{H} 4$ were not as readily identified.

As a graduate student, David Allis studied Drosophila development in Anthony Mahowald's lab at Indiana University and decided to pursue postdoctoral opportunities in gene regulation and chromatin biology. As Allis recently recounted to the JCI, "I got this tip from one of the postdocs in my PhD lab that there was this cool organism, Tetrahymena, if I was serious about chromatin biology. Tetrahymena is a single-celled organism that has two nuclei, the micronucleus and the macronucleus, and this individual in Rochester, New York, Martin Gorovsky, had pioneered the development of Tetrahymena as model and had worked out methods to not only grow the organism, but also to separate the two nuclei." Allis became interested in the differences between the micronucleus, which is transcriptionally silent in the vegetative state, and the transcriptionally active macronucleus, which controls phenotypic output, and began to explore these organelles in more depth. "There were just striking differences between the two nuclei. It was like black and white. One of the things that really jumped out on the appropriate gel was very maximally acetylated histones in the macronucleus and, as far as I could tell, completely unacetylated, or certainly hypoacetylated, histones coming out of the micronucleus." Allis continued work on the Tetrahymena model and sought to identify histone-modifying proteins. Specifically, the focus was on enzymes important for histone acetylation, which was a known marker of transcriptionally active chromatin. In 1996, the Allis group reported the isolation and characterization of a histone acetyltransferase (HAT) from Tetrahymena with homology to yeast Gcn5p, a yeast protein required for full transcriptional activation (7). The use of Tetrahymena was critical for the identification of HAT. According to Allis, "It was a Herculean effort on the part of Jim Brownell. Two hundred liters of Tetrahymena ultimately goes to two gel lanes, to a blot where the band, or the hopeful band, is cut out and sent off for sequencing."

Other groups were targeting the enzymes responsible for removing histone acetyl groups. Yoshida et al. identified a compound that prevented the removal of acetyl groups from histones, but not the target of this compound (8). Then, a month after Allis and Brownell published their study on HAT, Taunton, Hassig, and Schreiber reported the identification of a mammalian histone deacetylase (HDAC) with homology to the yeast transcriptional corepressor Rpd3p (9). The cumulation of this work fully supported histone modification as a fundamental regulator of gene expression. No longer could histones be viewed solely as DNA packaging.

\section{A field of study takes off}

Now that histones could no longer be viewed as passive participants in gene expression, there was great interest in further understanding how histones were modified, the enzymes responsible for the modifications, how these modifications were interpreted by the transcriptional machinery, and other factors. In addition to the seminal studies discussed above, the Allis and Grunstein labs have continually provided new insights into the regulators of histone and chromatin modification and both have led the charge in the field. It is now clear that histone modificationdependent regulation of the genome is an essential process that allows for precise control of gene expression among the diverse populations of cells that share identical genomes in an individual organism.

\section{Leaving a mark}

Allis and Grunstein both began their studies of histone and chromatin biology based on their interest in the science. When recently asked his advice on choosing a research path, Grunstein remarked, "The answer is you follow your nose. I certainly would not have predicted that regulation of transcription by histones would be so widespread. Honestly, I think there's a certain amount of luck, seren- dipity, and actual background that goes into how the field has developed. I am also convinced that your failures are as important as your successes in deciding your direction." Allis also shared a similar sentiment with the JCI: "I'm really a lab-centric person. What really drives me is the science and then, honestly, the people. You should do the research - at least I think - because you find that it's really a fundamentally interesting question. For me, that happened to center itself, a long time ago, around what are these histone proteins all about?"

Dysfunctional regulation of histone modification is now recognized as a driver of many diseases and adverse phenotypes, including multiple cancers, obesity, neurological disease, and X-linked syndromes; therefore, strategies to target HATs, HDACs, and other chromatin modifications are being explored for a myriad of diseases. Moreover, specific histone modifications can be useful biomarkers of disease progression and treatment response. Both Allis and Grunstein considered pursuing medicine early in their careers: however, they were drawn to the bench and inspired by excellent mentors to pursue research careers. This year's Lasker Basic Medical Research Award recognizes their work, which ultimately has helped lay the foundation for the identification of links between histone alterations and disease. Allis and Grunstein both noted that this recognition would not have happened without the fantastic individuals who have come through their labs. As for the future of the field, Grunstein told the JCI, "The field has certainly matured, but there's so much more to do that's exciting. For example, there are histone modifications associated with human tumor metastasis, and figuring out how histone modifications affect the progression of a tumor has got to be a new frontier." For Allis, "We have been fortunate to even have some of our molecular work remotely spin into potential therapeutic opportunities. I've had the opportunity, sadly, to meet some of these families who have lost children to diffuse intrinsic pontine glioma, a severe childhood brain cancer linked to point mutations within histone genes. It's a real inspiration to keep going and see what else we can learn to provide even a ray of hope for future treatment."

\section{Corinne L. Williams}


1. Kornberg RD, Lorch Y. Twenty-five years of the nucleosome, fundamental particle of the eukaryote chromosome. Cell. 1999;98(3):285-294.

2. Wu J, Grunstein M. 25 years after the nucleosome model: chromatin modifications. Trends Biochem Sci. 2000;25(12):619-623.

3. Lorch Y, LaPointe JW, Kornberg RD. Nucleosomes inhibit the initiation of transcription but allow chain elongation with the displacement of histones. Cell. 1987;49(2):203-210.

4. Allfrey VG, Faulkner R, Mirsky AE. Acetylation and methylation of histones and their possible role in the regulation of rna synthesis. Proc Natl Acad Sci U S A. 1964;51:786-794.

5. Han M, Grunstein M. Nucleosome loss activates yeast downstream promoters in vivo. Cell. 1988;55(6):1137-1145.

6. Durrin LK, Mann RK, Kayne PS, Grunstein M. Yeast histone $\mathrm{H} 4 \mathrm{~N}$-terminal sequence is required for promoter activation in vivo. Cell. 1991;65(6):1023-1031.

7. Brownell JE, et al. Tetrahymena histone acetyl- transferase A: a homolog to yeast Gcn5p linking histone acetylation to gene activation. Cell. 1996;84(6):843-851.

8. Yoshida M, Kijima M, Akita M, Beppu T. Potent and specific inhibition of mammalian histone deacetylase both in vivo and in vitro by trichostatin A. J Biol Chem. 1990;265(28):17174-17179.

9. Taunton J, Hassig CA, Schreiber SL. A mammalian histone deacetylase related to the yeast transcriptional regulator Rpd3p. Science. 1996;272(5260):408-411. 